\title{
Performance of optimized atom-centered potentials for weakly bonded systems using density functional theory
}

\author{
O. Anatole von Lilienfeld, Ivano Tavernelli, and Ursula Rothlisberger* \\ Ecole Polytechnique Fédérale de Lausanne (EPFL), Laboratoire de Chimie et Biochimie Computationelle, \\ CH-1015 Lausanne, Switzerland \\ Daniel Sebastiani \\ Max-Planck-Institut für Polymerforschung, D-55128 Mainz, Germany
}

(Received 12 January 2005; published 27 May 2005)

\begin{abstract}
Recently, we have introduced a scheme for optimizing atom-based nonlocal external potentials within the framework of density functional theory (DFT) in order to systematically improve the description of molecular properties [Phys. Rev. Lett. 93, 153004 (2004); J. Chem. Phys. 122, 014113 (2005)]. In this study, we investigate a small library of dispersion-corrected atom-centered potentials (DCACP's) for C, Ar, $\mathrm{Kr}$, and $\mathrm{Br}$. To this end, we calibrate DCACP's in order to reproduce the equilibrium distance and binding energy of MP2 potential energy surfaces of the weakly bonded homodimers $\mathrm{Ar}_{2}, \mathrm{Kr}_{2}$, and $\left(\mathrm{Br}_{2}\right)_{2}$. In all cases studied, using DFT with the generalized gradient approximation functional BLYP and the DCACP's, the influence of dispersion forces on equilibrium and transition-state geometries, interaction energies, and transition barriers can be reproduced in good agreement with MP2 calculations and without any significant increase in computational cost. The transferability of the DCACP's to other systems is assessed by addressing various weakly bonded complexes. We investigate (i) ideal van der Waals clusters of the type $\operatorname{Ar}_{n} \operatorname{Kr}_{m}(\forall n, m=\{0,1,2,3,4\}$ and 2 $\leqslant n+m \leqslant 4$ ), (ii) the effect of DCACP's on covalent bonds and conformers of the hydrocarbon molecule cyclooctatetraene which features a system of $\pi$ bonds, and (iii) the competition of simultaneous electrostatic and dispersion forces for the equilibrium structure and transition states of the hydrogen bromide dimer $(\mathrm{HBr})_{2}$. In all cases, the performance of the DCACP's to these extended set of systems is remarkably good.
\end{abstract}

DOI: 10.1103/PhysRevB.71.195119

PACS number(s): 31.15.Ew, 36.40.Mr, 87.15.Kg

\section{INTRODUCTION}

Weak long-range dispersion forces have a purely quantum origin: they represent the attractive interaction between selfinduced instantaneous dipole moments of isolated groundstate electron distributions. ${ }^{1}$ These forces are of crucial importance in nature because they influence many fundamental intermolecular and intramolecular phenomena such as, for example, the interactions between rare gas atoms, surface adsorptions, melting and boiling points, heats of sublimation, hydrophobic interactions, solvation effects, conformational properties of macromolecules (for instance, the tertiary and quaternary structures of biomolecules such as proteins or DNA), molecular recognition, the packing of molecular crystal, intercalation of drugs into DNA, and $\pi-\pi$ stacking effects, to name only a few. Recently, it has been shown that also macroscopic phenomena, such as the adhesion of gecko setae on surfaces, can be attributed solely to dispersion interactions. $^{2-4}$ However, while some density functional theory (DFT) exchange-correlation potentials show spurious binding for van der Waals (vdW) complexes, it is not yet generally possible to describe correctly vdW interactions within DFT using the local density approximation (LDA), the generalized gradient approximation (GGA), or even the-on average more accurate-hybrid exchangecorrelation functionals. ${ }^{5-10}$ In view of the ubiquitous nature of these forces the incorrect description of weak long-range interactions constitutes a serious failure of many conventional approximations to the exact exchange-correlation potential in DFT.
The introduction of nonlocality into a "vdW functional" as proposed in Refs. 11-13 or by electron density partitioning ${ }^{14}$ can abolish this deficiency but usually implies the arbitrary assignment of molecular fragments. Alternatively, Misquitta et al. ${ }^{15}$ and Kohn et al. ${ }^{16}$ proposed more general schemes which include dispersion in DFT but only at increased computational cost. As a consequence, empirical atom-atom-based correction terms have been proposed and assessed $^{8,17}$ using $C_{6}$ coefficients in the London $C_{6} / r^{6}$ asymptotic expression for the dispersion energy. Besides the fact that again fragments need to be assigned, these simple empirical corrections suffer from the fact that for each pair of fragments individual damping functions have to be identified to allow for the correct repulsive short-range behavior. Furthermore, preliminary calculations and assessments of appropriate $C_{6}$ coefficients are necessary, requiring, for instance, computationally more demanding time-dependent DFT calculations. ${ }^{18}$ Moreover, the electronic structure remains uncorrected by the use of this pair-potential-based correction.

Recently, we proposed within the framework of firstprinciples electronic structure calculations to optimize effective atom-centered potentials with respect to molecular references. ${ }^{19,20}$ The scheme was successfully applied to cure the lack of dispersion forces in standard DFT exchangecorrelation functionals. In analogy to the effective core potential (ECP) approach, the functional form of our atom centered potentials was chosen to be identical to the analytic pseudopotential by Goedecker and co-workers, ${ }^{21,22}$ but of a different range. These Gaussian-based norm-conserving 
dual-space pseudopotentials are separable and nonlocal. In the case of ECP generation their parameters are obtained for each atom by iteratively minimizing a penalty functional, which expresses the deviations of ECP-generated KohnSham (KS) valence orbitals from their (relativistic) allelectron counterparts. For our purposes, we have generalized this approach to molecules. We have chosen to retain the functional form of the ECPs for the following reasons: (i) the analytical form allows a rigorous differential approach for their optimization, (ii) the nonlocal form can inherently cast the nonlocal character of dispersion forces, (iii) no significant additional computational cost emerges upon the extension, and (iv) it is user friendly to include dispersion forces in DFT calculations by simply adding calibrated parameters to an ECP file. Hence, within our approach we include the molecular point of view into the design of an optimized atom-centered potential (OACP).

The OACP's obtained in such a way can improve the total Hamiltonian of the specific level of theory-if a suitable molecular reference can be defined for their calibration. For DFT as the electronic structure method, one can make use of density functional perturbation theory to fit the OACP's. ${ }^{20} \mathrm{In}$ particular, in order to remedy the failure of most GGA DFT calculations to describe London dispersion forces, we proposed to calibrate "dispersion-corrected" atom centered potentials (DCACP's). ${ }^{19}$ Specifically, our extended KS Hamiltonian reads $\hat{\mathcal{H}}_{\mathrm{ext}}^{\mathrm{KS}}=\hat{\mathcal{H}}^{\mathrm{KS}}+\sum_{\mathrm{I}} V_{\mathrm{I}}^{\mathrm{SG}} \mathrm{G}_{l+1}$. Where the additional nonlocal potential $V_{\mathrm{I}}^{\mathrm{SG}_{l+1}}$ centered on every atom I corresponds to an additional angular momentum channel $l+1$ with the same functional form as the ECP by Goedecker et al. ${ }^{21}$ $V_{\mathrm{I}}^{\mathrm{SG}_{l+1}}$ needs to be "dispersion calibrated" for every atom type with the help of the potential energy surface (PES) of a suitable, typically homonuclear, vdW complex as a reference system. The interference between the DCACP's and ordinary ECP's is minimized by the fact that they act at different ranges in space: the ECP's are strongly limited to the atomic core regions of the molecule while the DCACP's act at longer range. Furthermore, in order to describe weakly bonded systems the magnitude of the additional correcting potential can be kept very small.

The purpose of this article is to provide a small library of DCACP's (for C, Br, Ar, and $\mathrm{Kr}$ ), which can be used within BLYP DFT, and to assess their transferability to a variety of geometries, systems, and interaction types. To this end, we follow a simple procedure to minimize a given penalty with respect to a reference. As a reference we compute largebasis-set MP2 interaction energy curves for typically homonuclear supermolecular $\mathrm{vdW}$ complexes. In the case of DCACP's, we have chosen an intuitive functional form for the penalty: namely, the interaction energy and ionic forces at equilibrium distance.

In order to assess the transferability we compute with BLYP DFT other complexes using these calibrated DCACP's without any further tuning. We compare our DFT results to corresponding $a b$ initio calculations at the same level of theory as the data used for calibration. The choices of (a) the specific form of the penalty, (b) to use the GGA functional BLYP, and (c) to use MP2/aug-cc-pVTZ calculations as a reference are arbitrary. This combination seems to be reason- able because the penalty is simple and BLYP and MP2 are well-established methods. However, we do not claim that this combination is optimal. The same procedure could equally well be applied in the context of any other penalty, exchange-correlation functional, or arbitrarily exact reference. However, in this study, we consider reference calculations at the MP2 level of theory to be a reasonable compromise between accuracy and computational cost. Highaccuracy methods like coupled cluster theory or selfconsistent-field (SCF) response methods (e.g., coupled perturbed Hartree-Fock calculations) are desirable as benchmarks. But unfortunately, for larger systems, theoretical treatments beyond MP2 rapidly become computationally intractable even as references.

We calibrate DCACP's for the atoms $\mathrm{Ar}, \mathrm{Kr}$, and $\mathrm{Br}$ for the reference systems $\mathrm{Ar}_{2}, \mathrm{Kr}_{2}$, and $\left(\mathrm{Br}_{2}\right)_{2}$ and we use the previously introduced carbon DCACP which was calibrated for the benzene dimer $\left(\mathrm{C}_{6} \mathrm{H}_{6}\right.$ in its sandwich conformation). ${ }^{19}$ The transferability and in particular the scaling of properties with system size were tested systematically by studying the equilibrium structures of all possible heteronuclear and homonuclear clusters of the $\mathrm{Ar}$ and $\mathrm{Kr}$ dimers, trimers, and tetramers. For the tetramers, we also investigate the description of planar transition states (TS's) on the PES. We have chosen the complexes of $\mathrm{Ar}$ and $\mathrm{Kr}$ because interactions between noble gases are solely due to dispersion interactions and their calculation represents thus an ideal benchmark. We also assess the performance of the DCACP's to describe intramolecular London dispersion forces for a system which is dominated by the steric effects of covalent bonds: the apolar organic molecule cyclooctatetraene (COT). The structure of COT and its conformational changes have been attracting the attention of scientists since a first NMR study in $1962 .{ }^{23}$ It is also used to study Hückel rules or Jahn-Teller effects, a precursor in the synthesis of semibullvalene, and as a quencher in laser devices. ${ }^{24-27} \mathrm{We}$ investigate here if within BLYP DFT the DCACP's can account for a better description of the relative energies of different conformers and if they perturb the covalent bonding. Finally, we consider a "mixed" system in which covalent chemical bonds, strong electrostatic interaction (dipoledipole), hydrogen bonding, and dispersion effects occur simultaneously. Such a mixed system allows us to test if our approach to include dispersion forces deteriorates in some respect the description of the other predominant interactions. This is of interest because hydrogen bonding and permanent dipole-dipole interactions act in a similar range as the London forces. For this purpose, we have chosen the hydrogen bromide dimer as a test case. $(\mathrm{HBr})_{2}$ is an ideal representative for the simultaneous presence of all these interactions. We study the equilibrium structure as well as several TS's on the PES.

\section{METHODS AND COMPUTATIONAL DETAILS}

\section{A. Theory}

The basic idea of our approach has been introduced in Refs. 19 and 20. It deals with the iterative minimization of a penalty functional $\mathcal{P}$ by variational tuning of adjustable pa- 
rameters of analytic atom-centered effective potentials which are of the same form as the pseudopotentials of Goedecker $e t$ $a l .{ }^{21}$ The way the penalty functional is designed determines which molecular property will be optimized. It usually penalizes the deviations of a selected physical property-e.g., ionic forces-computed with a given set of values for the ECP parameters (which we denote by $\sigma_{i}$ ), with respect to a reference value which can be computed at any level of theory. Thus, the minimization of $\mathcal{P}\left(\left\{\sigma_{i}\right\}\right)$ (with respect to any observable that can be expressed via the KS orbitals of the system) allows us to approach as much as possible an arbitrary reference-within the limits of the chosen functional form of the ECP approach.

The analytic pseudopotentials introduced by Goedecker and co-workers ${ }^{21,22}$ consist of a local part $V^{\mathrm{loc}}(\mathbf{r})$ and of nonlocal parts $V_{l}^{\mathrm{nl}}\left(\left\{\sigma_{i}\right\}, \mathbf{r}, \mathbf{r}^{\prime}\right)$ with specific projectors for each angular momentum channel $l$ :

$$
\begin{aligned}
V^{\mathrm{ECP}}\left(\mathbf{r}, \mathbf{r}^{\prime}\right)= & V^{(\mathrm{loc})}(\mathbf{r}) \delta\left(\mathbf{r}-\mathbf{r}^{\prime}\right)+\sum_{l} V_{l}^{(\mathrm{nl})}\left(\mathbf{r}, \mathbf{r}^{\prime}\right), \\
V^{(\mathrm{loc})}(\mathbf{r})= & \frac{-Z_{\mathrm{ion}}}{r} \operatorname{erf}\left[\frac{r}{r_{\mathrm{loc}} \sqrt{2}}\right]+\exp \left[-\frac{r^{2}}{2 r_{\mathrm{loc}}^{2}}\right] \\
& \times\left(C_{1}+C_{2} \frac{r^{2}}{r_{\mathrm{loc}}^{2}}+C_{3} \frac{r^{4}}{r_{\mathrm{loc}}^{4}}+C_{4} \frac{r^{6}}{r_{\mathrm{loc}}^{6}}\right), \\
V_{l}^{(\mathrm{nl})}\left(\mathbf{r}, \mathbf{r}^{\prime}\right)= & \sum_{m=-l}^{+l} Y_{l m}(\hat{\mathbf{r}}) \sum_{j, h=1}^{3} p_{l h}(r) h_{h j}^{l} p_{l j}\left(r^{\prime}\right) Y_{l m}^{*}\left(\hat{\mathbf{r}}^{\prime}\right),
\end{aligned}
$$

where $p_{l h}(r) \propto r^{l+2(h-1)} \exp \left[-r^{2} /\left(2 r_{l}^{2}\right)\right], \quad r=\left|\mathbf{r}-\mathbf{R}_{\mathrm{I}}\right| \quad$ on the position $\mathbf{R}$ of nucleus I, $\hat{\mathbf{r}}$ is the unit vector in the direction of $\mathbf{r}$, and $Y_{l m}$ denotes a spherical harmonic. $\left\{r_{\text {loc }}, C_{1}, C_{2}, C_{3}, C_{4}, h_{h j}^{l}, r_{l}, \ldots\right\}$ spanning the atom-specific parameter space $\left\{\sigma_{i}\right\}$, the dimensionality of it being determined by the largest angular momentum of the ECP. In this study, we choose to restrict the optimization of the DCACP's in all cases to the parameters belonging to a single additional (nonlocal) angular momentum channel. This is an arbitrary choice, which has been successful in our recent study on vdW complexes $;{ }^{19}$ however, the number of additional angular momenta and their parameters-i.e., the space in which $\mathcal{P}$ shall be minimized-is optional for the purposes of the optimization. For all our calibrations, we minimize the simple penalty functional $\mathcal{P}^{\text {disp }}\left(\mathbf{R}^{\text {ref }}\right)$,

$$
\mathcal{P}^{\text {disp }}\left(\mathbf{R}^{\text {ref }}\right)=\left|E^{\text {ref }}\left(\mathbf{R}^{\text {ref }}\right)-E\left(\mathbf{R}^{\text {ref }}\right)\right|^{2}+\sum_{\mathrm{I}}^{N_{\text {ions }}} w_{\mathrm{I}}\left|\mathbf{F}_{\mathrm{I}}\left(\mathbf{R}^{\text {ref }}\right)\right|^{2},
$$

by following the penalty derivatives $\left\{\mathrm{d} \mathcal{P}^{\mathrm{disp}}\left(\mathbf{R}^{\text {ref }}\right) / \mathrm{d} \sigma_{i}\right\}$. $E^{\text {ref }}\left(\mathbf{R}^{\text {ref }}\right)$ is the total potential energy of interaction computed with the reference method at the nuclear configuration $\mathbf{R}^{\text {ref }}$ which corresponds to the equilibrium geometry determined with the reference method (in our case MP2/aug-ccpVTZ). $E\left(\mathbf{R}^{\text {ref }}\right)$ corresponds to the total potential energy of the interaction computed with the DFT electronic structure theory (in our case with the BLYP exchange-correlation potential) for the same nuclear configuration as the reference. $\mathbf{F}_{\mathrm{I}}\left(\mathbf{R}^{\text {ref }}\right)$ are the ionic forces within the ECP electronic structure theory acting on the nuclei I, which have to vanish at the reference equilibrium position $\mathbf{R}^{\text {ref }}$. For every calibration, Eq. (4) is minimized by iterative variation of the parameters of the additional angular momentum channel. All MP2 calculations have been carried out using the aug-cc-pVTZ basis set ${ }^{28,29}$ as available in GAUSSIAN03. ${ }^{30}$ Sinnokrot et al. have shown for the benzene dimer that using this basis set a realistic estimate of the binding energy due to dispersion can be made. ${ }^{31}$ For all the MP2 calculations reported here, the counterpoise correction has been used to account for the basis set superposition error (BSSE). All DFT calculations have been carried out using the program CPMD, ${ }^{32}$ the xc-functional BLYP, ${ }^{33,34}$ pseudopotentials of Goedecker and co-workers, ${ }^{21,22}$ and a plane-wave cutoff of $150 \mathrm{Ry}$ in case of the rare gases Ar and $\mathrm{Kr}$ and $100 \mathrm{Ry}$ in case of the COT and the bromine systems (no DCACP's have been used for the hydrogen atoms). No BSSE correction is necessary for plane-wave calculations because the number of basis functions is not modified upon changing the number of atoms. For all geometry optimizations the residual tolerance for ionic forces has been set to 0.00045 for the MP2 or to 0.0005 a.u. for the DFT calculations.

\section{B. Rare gas atoms}

We have calibrated DCACP's for argon and krypton by minimizing Eq. (4) such that the depth and location of the minimum of the potential energy curves of the homonuclear dimers $\mathrm{Ar}_{2}$ and $\mathrm{Kr}_{2}$ are reproduced within an accuracy of $\approx 1 \mathrm{~J} / \mathrm{mol}$ (Fig. 2). To reach this accuracy, it turned out to be necessary for $\mathrm{Kr}$ to use a $2 \times 2$ matrix $h_{i j}^{f}$, while for Ar, a single $h_{11}^{d}$ coefficient was sufficient. The results of the calibration are presented in Fig. 2. After calibration the geometries of all possible dimers and trimers have been optimized at the BLYP DFT and at the MP2 level of theory. The equilibrium geometries of all the tetramers have only been obtained within DFT. Imposing a planar structure, also geometries have been optimized for all TS's on the PES of the tetramers.

\section{Cyclooctatetraene}

For carbon we have used the DCACP's which have been calibrated for the benzene dimer ${ }^{19,35}$ with $r_{p}=3.28, h_{11}^{p}$ $=-0.0035$. The equilibrium geometries of COT in its "tubshaped" $D_{2 d}$ and in its "crown-shaped" conformation were optimized using BLYP DFT. In the same way, also the $D_{4 h}$ TS geometry for the ring inversion has been obtained by imposing a planar structure. A view of COT in its TS, tub, and crown conformations is given in Fig. 1.

\section{Bromine compounds}

We have calibrated a DCACP for Br by minimizing Eq. (4) such that the depth and location of the minimum of the interaction energy of $\left(\mathrm{Br}_{2}\right)_{2}$ are reproduced. To this end, we have first optimized the geometry of $\mathrm{Br}_{2}$ using MP2. Keeping the intramolecular distance of the two molecular moieties 


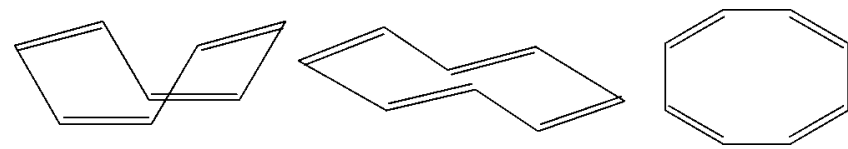

FIG. 1. Structures of cyclooctatetraene (COT) $\mathrm{C}_{8} \mathrm{H}_{8}$. Left: the "tub-shaped" $D_{2 d}$ global minimum conformation. Middle: the local minimum "crown-shaped" conformer. Right: the planar $D_{4 h}$ ring inversion transition state.

fixed, we have varied the intermolecular distance. Variation of the distance along a $D_{2 h}$-symmetry axis (all atoms in one plane) results in less binding energy than along a $C_{2 v}$-symmetry axis ("crossed" $\mathrm{Br}-\mathrm{Br}$ bonds). We have therefore chosen the latter for calibration purposes. The equilibrium geometry of the $(\mathrm{HBr})_{2}$ complex has been optimized using MP2 and DFT. In addition three TS structures for the hydrogen-bond isomerization have also been investigated. Their geometries have been taken from Ref. 36 .

\section{RESULTS AND DISCUSSION}

\section{A. Rare gas atoms}

For the homonuclear dimers $\mathrm{Ar}_{2}$ and $\mathrm{Kr}_{2}$ the MP2 PES curves $\left(E^{\text {int }}=E^{\text {dimer }}-2 E^{\text {monomer }}\right)$ as a function of interatomic distance, the initial BLYP DFT curves, and the final DCACP BLYP DFT curves are shown in Fig. 2. The resulting values for the DCACP's are $r_{d}=2.86, h_{11}^{d}=-0.002$ for $\mathrm{Ar}$ and $r_{f}$ $=3.61, h_{11}^{f}=-0.0015, h_{22}^{f}=0.004$ for Kr. Our MP2 results $\left(-0.98 \mathrm{~kJ} / \mathrm{mol}\right.$ and $-1.42 \mathrm{~kJ} / \mathrm{mol}$ for $\mathrm{Ar}_{2}$ and $\mathrm{Kr}_{2}$, respectively) are in good agreement with corresponding results obtained from recent literature. ${ }^{37}$ As expected, the initial BLYP DFT curves show a purely repulsive behavior in both cases. The calibrated DCACP BLYP DFT curves reproduce accurately the minimum of the reference curves, however in the case of $\mathrm{Kr}$ three parameters need to be optimized, whereas for Ar two parameters are sufficient. While also the repulsive part of the reference curves is remarkably well reproduced, the characteristic asymptotic $1 / r^{6}$ tail of dispersion interac-

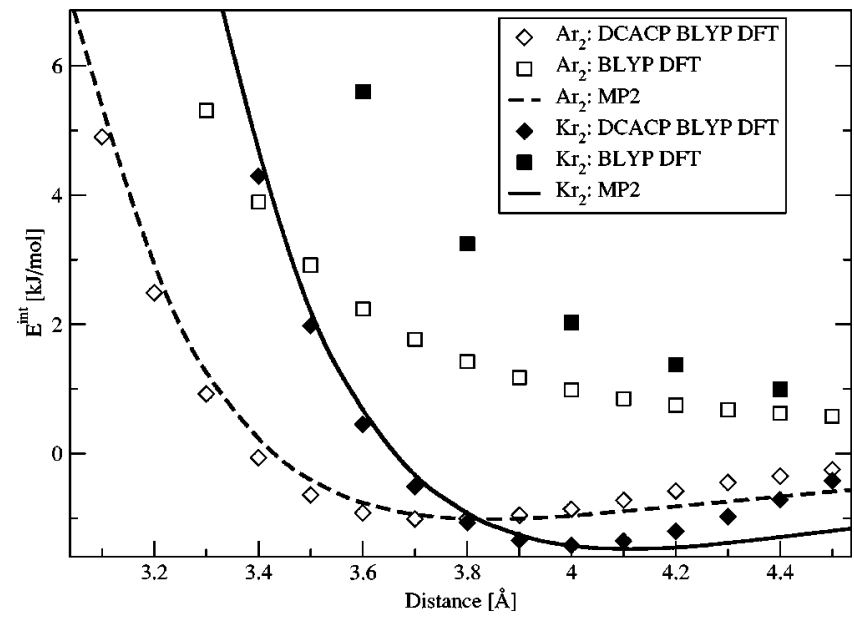

FIG. 2. Calibration of $\mathrm{Ar}$ and $\mathrm{Kr}$ for homonuclear dimers: the total energy of interaction $\left(E^{\text {int }}=E^{\text {dimer }}-2 E^{\text {monomer }}\right)$ as a function of interatomic distance.
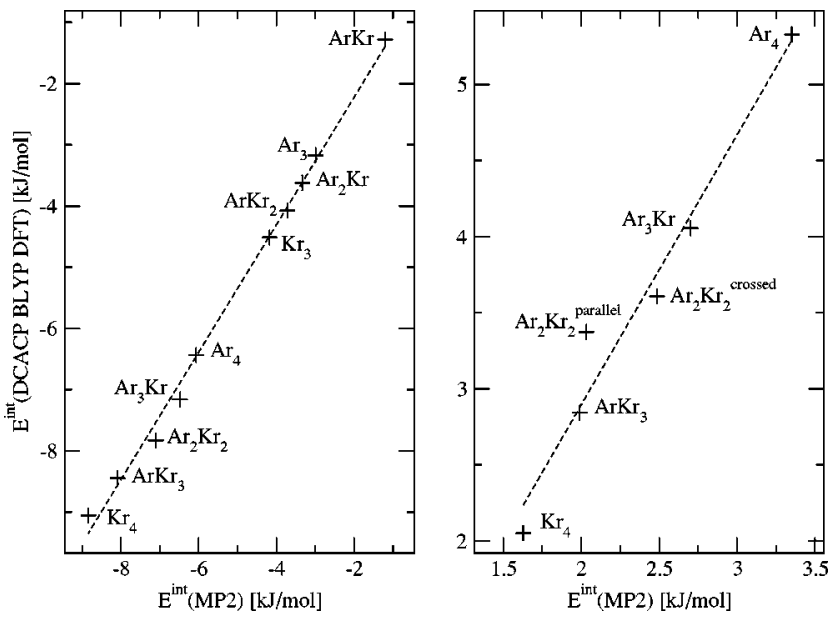

FIG. 3. Left panel: correlation of the interaction energies for equilibrium structures of $\mathrm{Ar}$ and $\mathrm{Kr}$ dimers, trimers, and tetramers (correlation coefficient: 0.998). Right panel: correlation of the interaction energies of planar transition states for $\mathrm{Ar}$ and $\mathrm{Kr}$ tetramers (correlation coefficient: 0.980 ). The total energy of interaction $\left(E^{\text {int }}=E^{\text {dimer }}-2 E^{\text {monomer }}\right)$ has been obtained within BLYP DFT using DCACP's and within MP2 using the counterpoise correction. For the dimer and trimers the geometry is optimized within DFT and MP2; for the tetramers the geometry is optimized only using DFT. The MP2 interaction energies of the tetramers have been obtained at the DFT geometry. The root-mean-square deviations (rms $\left.=\left\{1 / N \Sigma_{i}^{N}\left[E_{i}^{\text {int }}(\mathrm{MP} 2)-E_{i}^{i n t}(\mathrm{DFT})\right]^{2}\right\}^{1 / 2}\right)$ of the equilibrium and transition-state geometry are $0.41 \mathrm{~kJ} / \mathrm{mol}$ and $1.27 \mathrm{~kJ} / \mathrm{mol}$, respectively.

tion is less well described. However, this is not surprising since the $1 / r^{6}$ behavior is at the moment not included in the penalty functional and since the nonlocal functional form of the DCACP's does not necessarily imply a correct dissociation behavior. We have also investigated the effect of the use of DCACP's on the electronic structure for the case of $\mathrm{Ar}_{2}$. The effect is negligible: upon inclusion of DCACP's, the integrated electron density difference with respect to the MP2 density is reduced by $10^{-6}$ a.u.

Using DCACP BLYP DFT, the total energies of interaction for equilibrium geometries of all possible $\mathrm{Ar}$ and $\mathrm{Kr}$ heterodimer, trimers, and tetramers correlate very well with MP2 calculations (left-hand side of Fig. 3) within a rootmean-square deviation of only $0.41 \mathrm{~kJ} / \mathrm{mol}$. Also the interaction energies of the planar TS structures of all tetramers correlate well with corresponding MP2 values (right-hand side of Fig. 3) with a root-mean-square deviation of $1.27 \mathrm{~kJ} / \mathrm{mol}$. The scaling for the increasing van der Waals energy for an increasing number of $\mathrm{Kr}$ atoms over the number of Ar atoms is well reproduced. There are two possible planar TS's for the $\mathrm{Ar}_{2} \mathrm{Kr}_{2}$ tetramer: "crossed" and "parallel" arrangements, the result for the parallel configuration shows the largest deviation $(\approx 0.25 \mathrm{~kJ} / \mathrm{mol})$ but is still very accurate with respect to MP2. The slight increase of the rootmean-square deviation when going from equilibrium structures to TS's might be due to the fact that for the TS's the MP2 energies result from structures which have been only optimized using DCACP BLYP DFT or also due to the general tendency of BLYP to underestimate activation energies. 
TABLE I. Calculated transition barriers for the ring inversion of the tub-shaped cyclooctatetraene (COT) via a $D_{4 h}$ planar transition state and energy differences between tub-shaped and crown-shaped conformers (TC's). Every structure has been geometry optimized; for the transition states a planar geometry has been imposed. All values are in $\mathrm{kJ} / \mathrm{mol}$.

\begin{tabular}{ccccccc}
\hline \hline System & DFT $^{\mathrm{a}}$ & DCACP DFT $^{\mathrm{a}}$ & DFT $^{\mathrm{b}}$ & $\mathrm{CAS}^{\mathrm{c}}$ & $\mathrm{G}^{\mathrm{d}}$ & $\mathrm{CAS}^{\mathrm{e}}$ \\
\hline Barrier & 39.3 & 46.4 & 48.2 & 45.6 & 57.4 & 45.6 \\
TC & 55.3 & 53.6 & - & - & - & 58.0 \\
\hline \hline
\end{tabular}

${ }^{\mathrm{a} B L Y P}$ DFT.

${ }^{b}$ B3LYP DFT results from Ref. 40.

${ }^{\mathrm{C}} \mathrm{CASSCF}$ results from Ref. 38 .

${ }^{\mathrm{d}} \mathrm{G} 2$ (MP2) results from Ref. 39.

${ }^{\mathrm{e}} \mathrm{CAS}$ results from Ref. 25 .

\section{B. Cyclooctatetraene}

The energy barrier for the conformational change leading to a ring inversion of COT has been determined by computing the energy difference between the conformational global minimum of COT in its tub-shaped $D_{2 d}$ conformation and its planar $D_{4 h}$ geometry. As presented in Table I, the DCACP BLYP DFT calculations yield a value which is significantly closer to CAS (Refs. 25 and 38) or MP2 (Ref. 39) estimates taken from the literature than the BLYP DFT value. The energetically less favorable crown-shaped conformer has likewise been geometry optimized and its relative total energy is compared to literature values (Table I). The results indicate that the use of DCACP's improves substantially the description of conformational barriers and that the effect of intramolecular dispersion interactions is not negligible for conformational processes. Furthermore, a certain degree of transferability with respect to the atomic state of hybridization in the molecule is demonstrated since opposed to benzene COT does not obey the Hückel rule and is therefore not aromatic. However, for relative energy differences between local geometrical minima on the PES (tub-COT and crownCOT), the dispersion forces seem to cancel and therefore no improvement can be shown with respect to results in the literature.

\section{Bromine compounds}

Geometry optimization of $\mathrm{Br}_{2}$ at the MP2/aug-ccpVTZ level yields an interatomic equilibrium distance of $2.28 \AA$. The energies of interaction curves after and before the calibration of a DCACP for Br are shown in Fig. 4. The intramolecular distance has been kept fixed for the calibration and calculation of the potential energy curves. In contrast to the calibration of the rare gas atoms, for $\left(\mathrm{Br}_{2}\right)_{2}$ the tail as well as the repulsive part of the reference interaction curve is less well reproduced.

After calibration on $\left(\mathrm{Br}_{2}\right)_{2}$ a set of four different geometries of the $\mathrm{HBr}$ dimer has been identified. Using once BLYP DFT and once DCACP BLYP DFT, we have optimized the geometry of the equilibrium structure (EQ) of $(\mathrm{HBr})_{2}$ as it is depicted on the left-hand side in Fig. 5. We have proceeded likewise with three TS's on the PES of the

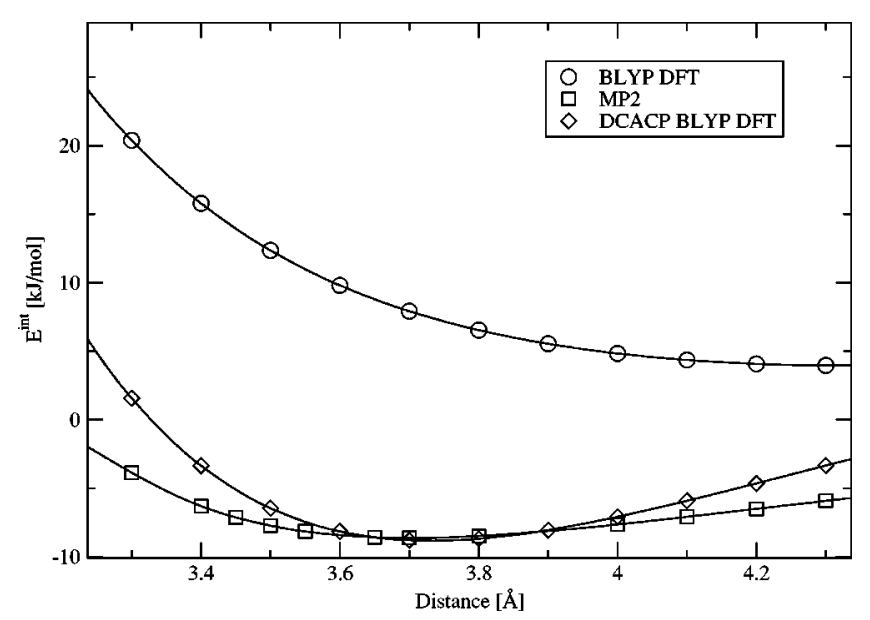

FIG. 4. Calibration of $\mathrm{Br}$ in the $\left(\mathrm{Br}_{2}\right)_{2}$ complex: the total energy of interaction $\left(E^{\text {int }}=E^{\text {dimer }}-2 E^{\text {monomer }}\right)$ as a function of the distance between two $\mathrm{Br}_{2}$ molecules.

HBr dimer: (i) The TS for the exchange of the intramolecular proton as it is depicted on the right-hand side in Fig. 5 (TS1). TS1 has been geometry optimized while keeping the $\mathrm{Br}-\mathrm{Br}$ distance fixed at $4.07 \AA$ and keeping both $\mathrm{HBrBr}$ angles fixed at $45^{\circ}$ (ii) The linear $[\mathrm{H}-\mathrm{Br} \cdot \mathrm{Br}-\mathrm{H}] \mathrm{TS}$ has been optimized by keeping the $\mathrm{Br}-\mathrm{Br}$ distance at $4.02 \AA$ and imposing linearity (TS2). (iii) Another linear TS $[\mathrm{Br}-\mathrm{H} \cdots \mathrm{Br}-\mathrm{H}]$ has been optimized by keeping the $\mathrm{Br}$ - $\mathrm{Br}$ distance at $4.33 \AA$ and imposing likewise linearity (TS3). The fixed distances between the $\mathrm{Br}$ atoms for the TS's were taken from Ref. 36 .

For all obtained geometries the corresponding MP2 single point calculations have been carried out for comparison. The deviations of the resulting total interaction energies from MP2 calculations are presented in Table II. While the standard BLYP DFT calculations show a systematic deviation by roughly $4 \mathrm{~kJ} / \mathrm{mol}$ the DCACP BLYP DFT calculations compare significantly better and do no longer exhibit a systematic deviation. All the MP2 calculations carried out with the DCACP BLYP DFT geometries show more interaction than those carried out with the BLYP DFT optimized structures. As in the case of the tetramers of Ar and $\mathrm{Kr}$, also for $(\mathrm{HBr})_{2}$ the energy of interaction of the equilibrium structure compares with MP2 results better than the results for the TS's.

\section{CONCLUSIONS}

Using the standard GGA functional BLYP, we have presented an overview of DFT calculations for some vdW complexes with and without the dispersion correction. The

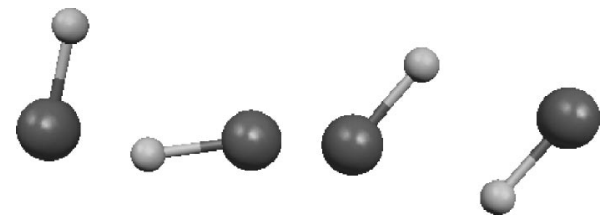

FIG. 5. Left: the $\mathrm{HBr}$ dimer in its equilibrium geometry (EQ): $\mathrm{Br}-\mathrm{Br}$ distance at $4.1 \AA \mathrm{A}, \mathrm{H}-\mathrm{Br}-\mathrm{Br}$ angle is $80^{\circ}$ or $10^{\circ}$, respectively. Right: the hydrogen exchange transition state with $45^{\circ}$ for both $\mathrm{H}-\mathrm{Br}-\mathrm{Br}$ angles (TS1) is presented. Both complexes are planar. 
TABLE II. Deviations of total BLYP DFT energies of interaction from MP2 results. The conformations of EQ, TS1, TS2, and TS3 are explained in the text and in Fig. 5. All values are in $\mathrm{kJ} / \mathrm{mol}$ and correspond to $E_{M P 2}^{\text {int }}-E_{D F T}^{\text {int }}$.

\begin{tabular}{cccrc}
\hline \hline Structure & EQ & TS1 & TS2 & TS3 \\
\hline DFT $^{\mathrm{a}}$ & -4.2 & -6.0 & -4.3 & -4.3 \\
DCACP DFT $^{\mathrm{b}}$ & -0.3 & -0.6 & 0.5 & -1.2 \\
\hline \hline
\end{tabular}

${ }^{\mathrm{a}} \mathrm{MP} 2$ results correspond to BLYP DFT geometry.

${ }^{\mathrm{b}} \mathrm{MP} 2$ results correspond to DCACP BLYP DFT geometry.

DCACP's have been obtained by calibration with respect to simple homonuclear weakly bonded reference systems and could successfully be applied to other systems without any further changes. Specifically, we have described equilibrium structures and transition states of all rare gas dimers, trimers, and tetramers consisting of $\mathrm{Ar}$ and $\mathrm{Kr}$. Furthermore, we could show for conformers of COT that the use of DCACP's improves the description of a conformational barrier, while it does not affect the energy difference between local geometrical minima. The calculation of the interaction in the hydrogen-bonded hydrogen bromide dimer is improved con- siderably for the ground-state geometry as well as for transition states on the PES. In our previous study, ${ }^{19}$ we could also show an accurate description for argon-benzene, benzene-benzene, and graphite sheets.

In total, we believe that our results suggest that dispersion forces can be reliably predicted in a transferable and general fashion within DFT using DCACP's. We conclude that for many systems of chemical interest the predictive power of GGA DFT can be enforced substantially when using DCACP's while leaving the computational cost constant. Work is in progress to test the DCACP's in combination with other exchange correlation functionals than BLYP, to explicitly include the correct short-range repulsive and the asymptotic $1 / r^{6}$ behavior into the penalty functional, to optimize the functional form of the DCACP, and to establish a comprehensive library of DCACP's for every atom type.

\section{ACKNOWLEDGMENTS}

The authors thank G. Fichet, T. Wesolowski, and M. Parrinello for discussions. This work was supported by the Swiss National Science Foundation, Grant No. 510573.
*Electronic address: ursula.roethlisberger@epfl.ch; URL: http:// lcbcpc21.epfl.ch

${ }^{1}$ D. P. Craig and T. Thirunamachandran, in Molecular Quantum Electrodynamics (Dover, Mineola, NY, 1998).

${ }^{2}$ K. Autumn, M. Sitti, Y. A. Liang, A. M. Peattie, W. R. Hansen, S. Sponberg, T. W. Kenny, R. Fearing, J. N. Israelachvili, and R. J. Full, Proc. Natl. Acad. Sci. U.S.A. 99, 12252 (2002).

${ }^{3}$ K. Autumn, Y. A. Liang, S. T. Hsieh, W. Zesch, W. P. Chan, T. W. Kenny, R. Fearing, and R. J. Full, Nature (London) 405, 681 (2000).

${ }^{4}$ C. Gay and L. Leibler, Phys. Rev. Lett. 82, 936 (1999).

${ }^{5}$ W. Koch and M. C. Holthausen, A Chemist's Guide to Density Functional Theory (Wiley-VCH, New York, 2002).

${ }^{6}$ S. Kristyán and P. Pulay, Chem. Phys. Lett. 229, 175 (1994).

${ }^{7}$ J. M. Pérez-Jordá and A. D. Becke, Chem. Phys. Lett. 233, 134 (1995).

${ }^{8}$ E. J. Meijer and M. Sprik, J. Chem. Phys. 105, 8684 (1996).

${ }^{9}$ T. van Mourik and R. J. Gdanitz, J. Chem. Phys. 116, 9620 (2002)

${ }^{10}$ X. Wu, M. C. Vargas, S. Nayak, V. Lotrich, and G. Scoles, J. Chem. Phys. 115, 8748 (2001).

${ }^{11}$ Y. Andersson, D. C. Langreth, and B. I. Lundqvist, Phys. Rev. Lett. 76, 102 (1996).

${ }^{12}$ E. Hult, Y. Andersson, B. I. Lundqvist, and D. C. Langreth, Phys. Rev. Lett. 77, 2029 (1996).

${ }^{13}$ H. Rydberg, B. I. Lundqvist, D. C. Langreth, and M. Dion, Phys. Rev. B 62, 6997 (2000).

${ }^{14}$ T. A. Wesolowski and F. Tran, J. Chem. Phys. 118, 2072 (2003).

${ }^{15}$ A. J. Misquitta, B. Jeziorski, and K. Szalewicz, Phys. Rev. Lett. 91, 033201 (2003).

${ }^{16}$ W. Kohn, Y. Meir, and D. E. Makarov, Phys. Rev. Lett. 80, 4153 (1998).
${ }^{17}$ R. LeSar, J. Phys. Chem. 88, 4272 (1984).

${ }^{18}$ S. J. A. van Gisbergen, J. G. Snijders, and E. J. Baerends, J. Chem. Phys. 103, 9347 (1995).

${ }^{19}$ O. A. von Lilienfeld, I. Tavernelli, U. Rothlisberger, and D. Sebastiani, Phys. Rev. Lett. 93, 153004 (2004).

${ }^{20}$ O. A. von Lilienfeld, I. Tavernelli, U. Rothlisberger, and D. Sebastiani, J. Chem. Phys. 122, 14113 (2005).

${ }^{21}$ S. Goedecker, M. Teter, and J. Hutter, Phys. Rev. B 54, 1703 (1996).

${ }^{22}$ C. Hartwigsen, S. Goedecker, and J. Hutter, Phys. Rev. B 58, 3641 (1998).

${ }^{23}$ F. a. L. Anet, J. Am. Chem. Soc. 84, 671 (1962).

${ }^{24}$ M. J. S. Dewar, A. Harget, and E. Haselbach, J. Am. Chem. Soc. 91, 7521 (1969).

${ }^{25}$ J. L. Andrés, O. Castano, A. Morreale, R. Palmeiro, and R. Gomperts, J. Chem. Phys. 108, 203 (1998).

${ }^{26}$ H. E. Zimmerman and H. Iwamura, J. Am. Chem. Soc. 92, 2015 (1970).

${ }^{27}$ R. P. Frueholz and A. Kuppermann, J. Chem. Phys. 69, 3614 (1978).

${ }^{28}$ T. H. Dunning, Jr., J. Chem. Phys. 90, 1007 (1989).

${ }^{29}$ K. A. Peterson, D. E. Woon, T. H. Dunning, Jr., J. Chem. Phys. 100, 7410 (1994).

${ }^{30}$ M. J. Frisch et al., computer code GAUSSIAN 03, revision A.1, 2003.

${ }^{31}$ M. O. Sinnokrot, E. F. Valeev, and C. D. Sherrill, J. Am. Chem. Soc. 124, 10887 (2002).

${ }^{32} \mathrm{~J}$. Hutter et al., computer code CPMD, version 3.8, IBM Corp. and MPI-FKF Stuttgart, 1990-2003, http://www.cpmd.org

${ }^{33}$ A. D. Becke, J. Chem. Phys. 88, 2547 (1988).

${ }^{34}$ C. Lee, W. Yang, and R. G. Parr, Phys. Rev. B 37, 785 (1988).

${ }^{35}$ In our previous study (Ref. 19), we have erroneously indicated that we have added a $d$ channel to the ECP of carbon and an $f$ 
channel to the ECP of argon. In fact, we added a $p$ channel to carbon and a $d$ channel to argon.

${ }^{36}$ J. Castillo-Chará, A. L. McIntosh, Z. Wang, R. R. Lucchese, and J. W. Bevan, J. Chem. Phys. 120, 10426 (2004).

${ }^{37}$ P. Slavicek, R. Kalus, P. Paska, I. Odvarkova, P. Hobza, and A. Malijevsky, J. Chem. Phys. 119, 2102 (2003).
${ }^{38}$ O. Castano, R. Palmeiro, L. M. Frutos, and J. Luisandrés, J. Comput. Chem. 23, 732 (2002).

${ }^{39}$ T. M. Miller, A. A. Viggiano, and A. E. S. Miller, J. Phys. Chem. A 106, 10200 (2002).

${ }^{40}$ D. H. Paik, D. Yang, I. Lee, and A. H. Zewail, Angew. Chem., Int. Ed. Engl. 43, 2830 (2004). 